\title{
Positivity Bounds for the Y-ADM Mass Density
}

\author{
David Kastor ${ }^{(1)}$, Tetsuya Shiromizu ${ }^{(2,3,4)}$, Shinya Tomizawa ${ }^{(2)}$ and Jennie Traschen ${ }^{(1)}$ \\ (1) Department of Physics, University of Massachusetts, Amherst, MA 01003, US \\ ${ }^{(2)}$ Department of Physics, Tokyo Institute of Technology, Tokyo 152-8551, Japan \\ (3) Department of Physics, The University of Tokyo, Tokyo 113-0033, Japan and \\ ${ }^{(4)}$ Advanced Research Institute for Science and Engineering, Waseda University, Tokyo 169-8555, Japan
}

(Dated: June 29, 2018)

\begin{abstract}
Killing-Yano tensors are natural generalizations of Killing vectors to arbitrary rank anti-symmetric tensor fields. It was recently shown that Killing-Yano tensors lead to conserved gravitational charges, called Y-ADM charges. These new charges are interesting because they measure e.g. the mass density of a $p$-brane, rather than the total ADM mass which may be infinite. In this paper, we show that the spinorial techniques used by Witten, in his proof of the positive energy theorem, may be straightforwardly extended to study the positivity properties of the Y-ADM mass density for $p$-brane spacetimes. Although the resulting formalism is quite similar to the ADM case, we show that establishing a positivity bound in the higher rank Y-ADM case requires imposing a condition on the Weyl tensor in addition to an energy condition. We find appropriate energy conditions for spacetimes that are conformally flat or algebraically special, and for spacetimes that have an exact Killing vector along the brane. Finally we discuss our expression for the Y-ADM mass density from the Hamiltonian point of view.
\end{abstract}

PACS numbers: 04.50.+h 11.25.-w 11.27.+d

\section{INTRODUCTION}

Conserved gravitational charges are associated with the asymptotic symmetries of a spacetime. For example, the ADM mass and angular momentum correspond to asymptotic time translation and rotation Killing vectors respectively. In $D$ spacetime dimensions these charges are given by integrals over a $(D-2)$-sphere at spatial infinity. The electric charge carried by matter and black holes in the spacetime interior is, of course, also given by an integral over a $(D-2)$-sphere. Charge conservation allows both these integrals to be evaluated at an arbitrary time.

For $p$-branes this coincidence no longer holds. The ADM mass, in this case, is given by an integral over a cylinder $R^{p} \times S^{D-(p+2)}$ at transverse spatial infinity that encloses the entire brane - i.e. the integral includes directions parallel to the brane. A $p$-brane world-volume naturally couples to a $(p+1)$-form gauge potential. The electric charge of the brane is given by an integral over an $S^{D-(p+2)}$ that encloses only a single point on the brane world-volume - i.e. the integral excludes directions parallel to the brane. Charge conservation allows the ADM integral to be evaluated at an arbitrary time, while the electric charge integral may be evaluated at an arbitrary position along the brane in space or time.

Symmetry may be restored to the formulation of gauge and gravitational charges for $p$-branes by the introduction of new gravitational charges, called Y-ADM charges, associated with Killing-Yano tensors [1]. A Killing-Yano tensor is an antisymmetric tensor field of arbitrary rank that satisfies a natural generalization of Killing's equation [2]. A rank 1 Killing-Yano tensor is simply a Killing vector. It was shown in reference [1] that the AbbottDeser (AD) construction of the ADM charge associated with an asymptotic Killing vector [3] may be generalized to give a conserved charge associated with an asymptotic Killing-Yano tensor of higher rank. The resulting Y-ADM charge is given by an integral over an $S^{D-(p+2)}$ at transverse spatial infinity. Like the electric charge carried by a brane, it is independent of time and also independent of translations of the surface of integration parallel to the brane.

For a $p$-brane spacetime, we focus on the rank $(p+$ 1) asymptotic Killing-Yano tensor given by the antisymmetric product of translations in the directions parallel to the world-volume of the brane. For a particle this is just the asymptotic time translation Killing vector and the corresponding charge is the ADM mass. For a general $p$-brane, we call the corresponding charge the $Y$-ADM mass density, which we will denote by $\mathcal{M}$ below.

One very important result associated with the ADM mass is the positive energy theorem [4]. It is natural to ask if any sort of positivity result holds for the Y-ADM mass density $\mathcal{M}$ ? In this paper we investigate this question, making use of the spinor techniques of Witten [5] and Nester [6]. We focus on the rank 2 case corresponding to a 1-brane, or string.

Our central result is a spinorial boundary integral expression for the Y-ADM mass density $\mathcal{M}$. Assuming that the spatial slices have no interior boundaries, e.g. at horizons, Stokes theorem relates this boundary integral to a volume integral, whose integrand is the sum of three terms. The first of these terms involves the stress-energy tensor; the second term is a positive definite expression quadratic in the derivative of the spinor field; the third term involves certain components of the Riemann tensor. The first two terms are similar to those that appear in Witten's proof of the positive energy theorem [5], in which an energy condition is sufficient to ensure positivity of the mass. The third term, however, cannot be 
written directly in terms of the stress-energy tensor and - hence - an energy condition is not sufficient to imply positivity of $\mathcal{M}$. We discuss two types of further conditions under which positivity nevertheless holds: (i) If the spacetime is conformally flat or algebraically special and (ii) if the spacetime has an exact translational symmetry along the brane.

The paper is organised as follows. Section II presents various preliminaries - definitions and results relating to Killling-Yano tensors, transverse asymptotically flat spacetimes, and spinors. In Section III, we briefly review the construction of Y-ADM charges [1] using the techniques of Abbott and Deser [3. We show, in particular for the rank 2 case, that the Y-ADM mass density $\mathcal{M}$ is intrinsic in the sense that it depends only on quantities intrinsic to the codimension 2 slice transverse to the string - a result that has a close parallel in the rank 1 case, in the formula for the ADM mass. Our spinorial construction for Y-ADM charges is presented in Section IV. In Section V, we demonstrate positivity of the mass density $\mathcal{M}$ under the special conditions mentioned above. Section VI is a discussion of preliminary results on the Y-ADM mass density as a $p$-brane Hamiltonian and some concluding remarks.

\section{PRELIMINARIES}

\section{A. Killing-Yano Tensors}

A Killing-Yano tensor [2] is a rank $n$ rank antisymmetric tensor field $f^{a_{1} \cdots a_{n}}=f^{\left[a_{1} \cdots a_{n}\right]}$ satisfying

$$
\nabla_{\left(a_{1}\right.} f_{\left.a_{2}\right) a_{3} \cdots a_{n+1}}=0 .
$$

For rank $n=1$ this condition reduces to Killing's equation. Flat spacetime has the maximal number of KillingYano tensors of each rank. In this paper we will focus on the case of rank $n=2$. For flat spacetime in Cartesian coordinates, a basis for rank 2 Killing-Yano tensors is given by the translational tensors

$$
f=d x^{a} \wedge d x^{b},
$$

together with the rotational tensors

$$
f=x^{a} d x^{b} \wedge d x^{c}+x^{b} d x^{c} \wedge d x^{a}+x^{c} d x^{a} \wedge d x^{b} .
$$

\section{B. Transverse asymptotically flat spacetimes}

The notion of transverse asymptotic flatness in $D$ dimensions, as discussed in reference [7], is motivated by considering $p$-brane spacetimes. Such spacetimes become flat only as we approach transverse spatial infinity in the $D-(p+1)$ directions transverse to the brane; not by moving along the $p$ spatial directions parallel to the brane [14].
Write the full spacetime metric as $g_{a b}=\eta_{a b}+h_{a b}$, where $\eta_{a b}$ is the flat Minkowski metric. Let $\left(x^{\mu}, x^{I}\right)$ with $\mu=0,1, \ldots, p$ and $I=p+1, \ldots, D-1$ be Cartesian coordinates in the asymptotic region and let $r^{2}=\delta_{I J} x^{I} x^{J}$. Transverse asymptotic flatness requires that in the limit $r \rightarrow \infty$ the components of $h_{a b}$ fall-off as

$$
h_{\mu \nu}, h_{I J} \sim \mathcal{O}\left(\frac{1}{r^{D-(p+3)}}\right), \quad h_{\mu I} \sim \mathcal{O}\left(\frac{1}{r^{D-(p+2)}}\right) .
$$

Note, in particular, that cross terms between the directions tangent and transverse to the brane are required to fall off one power faster than the other components.

In this paper we will focus on the case of 1-branes, or strings. In addition to transverse asymptotically flat boundary conditions with $p=1$, we will assume that the spacetime interior can be foliated by codimension two spatial submanifolds, which we will think of as transverse to the brane. At infinity these spatial submanifolds are taken to be aligned with the $\left(x^{2}, \ldots, x^{D-1}\right)$ hyperplane. The spacetime metric may then be written as $g_{a b}=L_{a b}+$ $q_{a b}$, with $L_{a}^{b} q_{b c}=0$, where $q_{a b}$ is a Euclidean metric on the $(D-2)$ dimensional submanifolds and $L_{a}^{b}$ projects onto the directions normal to these submanifolds. It will also prove useful to be able to further split the metric as

$$
g_{a b}=-n_{a} n_{b}+y_{a} y_{b}+q_{a b}
$$

where $n^{a}$ and $y^{a}$ are normal vectors to the submanifolds satisfying $n_{a} n^{a}=-1, y_{a} y^{a}=1, n_{a} y^{a}=0$. At infinity, we take

$$
n^{a}=\left(\partial / \partial x^{0}\right)^{a}, \quad y^{a}=\left(\partial / \partial x^{1}\right)^{a} .
$$

The asymptotic Killing-Yano tensor used to define the Y-ADM mass density of a string in the $x^{1}$ direction has non-zero coomponents $f^{01}=-f^{10}=1$. At infinity, we can then also write

$$
f^{a b}=n^{a} y^{b}-y^{a} n^{b} .
$$

The construction of the Y-ADM mass density for a string takes place on a codimension two volume $V$ with the two normals $n^{a}$ and $y^{a}$, and metric $q_{a b}$. The embedding of $V$ in the full spacetime is described by the extrinsic curvature tensor $K_{a b}{ }^{c}=q_{a}{ }^{m} q_{b}{ }^{n} \nabla_{m} q_{n}{ }^{e}$. The indices $a, b$ are tangent to $V$, and the index $c$ is normal, i.e. $q_{c d} K_{a b}{ }^{c}=0$. The extrinsic curvature $K_{a b}{ }^{c}$ is symmetric on the indices $a, b$, a result that follows from Frobenius' Theorem. The Gauss-Codazzi relations, which will be used in subsequent calculations, are

$$
q_{a}{ }^{e} q_{b}{ }^{f} q_{c}{ }^{r} q_{s}^{d} R_{e f r}{ }^{s}[g]=R_{a b c}{ }^{d}[q]-K_{c a}{ }^{e} K_{b e}{ }^{d}+K_{c b}{ }^{e} K_{a}{ }^{d}
$$

The extrinsic curvature can be decomposed as $K_{a b}^{c}=$ $\alpha_{a b} n^{c}+\beta_{a b} x^{c}$, where $\alpha_{a b}=q_{a}^{c} q_{b}^{d} \nabla_{c} n_{d}$, and $\beta_{a b}=$ $-q_{a}^{c} q_{b}^{d} \nabla_{c} y_{d}$.

\section{Spinors and Killing-Yano tensors}

We are interested in extending Witten's spinorial techniques to study the positivity of Y-ADM charges. Hence, 
we assume the existence of a spinor field $\psi$ in the spacetime. At transverse spatial infinity we will assume that $\psi$ approaches one of the constant spinors $\psi^{(0)}$ of flat spacetime. We will make use of the quantity $f_{a b}=$ $-\psi^{\dagger} \gamma^{\hat{0} \hat{1}} \gamma_{a b} \psi$ below, where $\gamma^{\hat{0}} \equiv-n_{a} \gamma^{a}$ and $\gamma^{\hat{1}} \equiv x_{a} \gamma^{a}$. In the asymptotic limit, where $\psi$ is replaced by $\psi^{(0)}$, the tensor $f_{a b}$ is a Killing-Yano tensor. Note that we can only construct the translational Killing-Yano tensors (2) of the background flat spacetime in this way. The particular Killing-Yano tensor of interest to us (7) is of this form.

\section{Y-ADM CHARGES}

We begin by briefly reviewing the construction of YADM charges in transverse asymptotically flat spacetimes [1]. We will continue to focus on the case of rank 2 Killing-Yano tensors. The general case is considered in [1]. The construction is an extension of the Abbott and Deser construction of a conserved ADM charge associated with the Killing vectors of flat spacetime [3].

The construction begins by writing the metric in the form $g_{a b}=\eta_{a b}+h_{a b}$ throughout the spacetime. The difference $h_{a b}$ between the full metric and the flat metric may be large in the interior of the spacetime, but vanishes at the rates specified by Eqs. (4) near transverse spatial infinity. Let $f^{a b}$ be a Yano tensor for the background metric. Using the Bianchi identities and the defining properties of Yano tensors, one can construct a rank 2 antisymmetric tensor current $k^{a b}$ which is conserved with respect to the background derivative operator, i.e. $\nabla_{a}^{(0)} k^{a b}=0$. This current is given by

$$
k^{a b}=f^{a c} R_{c}^{L b}-f^{b c} R_{c}^{L a}-\frac{1}{2} f^{a b} R^{L}-\frac{1}{2} f^{c d} R_{c d}^{L a b},
$$

where $R_{c d}^{L a b}$, for example, is the linear term in the formal power series expansion of $R_{c d}^{a b}$ in $h_{a b}$.

Conservation of the 2 -form current $k$ can be rewritten as $d * k=0$, where $*$ indicates the Hodge dual with respect to the background flat metric. It then follows that there locally exists a 3 -form $l$, such that $* k=d * l$. One finds that the 3 -form $l$ is given by

$$
\begin{aligned}
l^{a b c}= & 3 \delta_{k}^{[a} \delta_{l}^{b} \delta_{m}^{c} \delta_{n}^{d]} f^{k l}\left(\bar{\nabla}^{m} h_{d}^{n}\right)-\frac{1}{4}\left(\bar{\nabla}^{[a} f^{b c]}\right) h_{d}^{n} \\
& +\frac{3}{4}\left(\bar{\nabla}^{d} f^{[a b}\right) h_{d}^{c]},
\end{aligned}
$$

As in the previous section, let $V$ be a spacelike, codimension two slice with normals $n^{a}$ and $x^{a}$. The conserved Y-ADM charge is then defined by the integral of the $(D-3)$-form $* l$ over $\partial V$ as $\partial V \rightarrow \infty$, i.e., over a closed, codimension two, section of spatial infinity,

$$
Q\left[f_{a b}\right]=\frac{1}{8 \pi} \int_{\partial V} * l,
$$

where we have used the notation $Q\left[f_{a b}\right]$ to denote the charge associated with an arbitrary asymptotic KillingYano tensor $f_{a b}$.

We now focus on the Y-ADM mass density $\mathcal{M}$, which means specifying the background Killing-Yano tensor $f^{a b}$ as in equation (7) and thereabove. In this case, since $\bar{\nabla}_{a} f^{b c}=0$, only the first term in equation (10) is nonzero. Substituting the identification (7) into this term and carrying out the anti-symmetrization then yields the expression for the Y-ADM mass density

$$
\mathcal{M}=\frac{1}{8 \pi} \int_{\partial V} d s_{a} q^{a d} q^{c b} \bar{\nabla}_{[b} \delta g_{d] c} .
$$

We can further specialize to Cartesian coordinates near infinity, giving the form

$$
\mathcal{M}=\frac{1}{8 \pi} \int_{\partial V} d s_{I}\left(\partial_{J} h^{I J}-\partial^{I} h_{J}^{J}\right)
$$

which is very similar to the usual formula for the ADM mass $M$, differing only in the higher codimension of the surface $V$ and the correspondingly smaller range of the indices summed over - here $I, J=2, \ldots, D-1$.

It is interesting to compare the values of the $\mathrm{Y}-\mathrm{ADM}$ mass density $\mathcal{M}$ and the ADM mass $M_{A D M}$. As noted above, if the $x^{1}$ direction tangent to the string is infinite in extent, then the ADM mass $M_{A D M}$ will itself be infinite. The ADM mass will be finite If we consider spacetimes such that $x^{1}$ compact. If we make the identification $x^{1} \equiv x^{1}+L$, then the ADM mass is given by

$$
\begin{aligned}
M_{A D M} & =\int_{0}^{L} d x^{1} \int_{\partial V} d s_{I}\left(\partial_{J} h^{I J}-\partial^{I} h_{J}^{J}-\partial^{I} h_{1}{ }^{1}\right) \\
& =L\left(\mathcal{M}-\mathcal{M}_{\text {scalar }}\right),
\end{aligned}
$$

where the scalar charge density $\mathcal{M}_{\text {scalar }}$ is defined to by the integral over $\partial_{V}$ of the final term in the integrand above.

It is, lastly, instructive to rewrite the expression for the Y-ADM mass density in terms of $\Lambda_{a b}$, the extrinsic curvature of $\partial V$ in $V$. One finds

$$
\mathcal{M}=\frac{1}{16 \pi} \int_{\partial V} d v\left(\Lambda_{I}^{I}-\Lambda_{(\mathrm{bg}) I}^{I}\right)
$$

where $\Lambda_{(\mathrm{bg})}^{I J}$ is the extrinsic curvature of $\partial V$ within $V$ evaluated with respect to the background flat metric. This expression has the same form as that given by Hawking and Horowitz for the ADM mass in reference [10], except that in their work the integral is over the boundary of a codimension one volume.

\section{SPINOR CONSTRUCTION OF THE Y-ADM MASS DENSITY $\mathcal{M}$}

In his proof of the positive energy theorem [5], Witten found an expression for the ADM mass boundary integral 
in terms of a spinor field. Stokes' theorem then provides an alternative volume integral expression for the ADM mass which, providing the spinor satisfies a certain Diractype equation on the spacelike hypersurface, is the sum of two terms. The first of these terms, involving the Einstein tensor, is positive provided the dominant energy condition is satisfied. The second term, quadratic in first derivatives of the spinor field, is manifestly positive and vanishes only in flat spacetime. Witten's formalism was later streamlined by Nester 6]

In this section, we develop a generalization of the Witten-Nester formalism that yields a spinor expression for the Y-ADM mass density $\mathcal{M}$ for a 1 -brane spacetime. The corresponding volume integral in this case is again the sum of two terms. The term quadratic in first derivatives of the spinor field is again positive definite. However, the analogue of the Einstein tensor term now has contributions from the un-contracted Riemann tensor as well. Positivity of these terms cannot be ensured by an energy condition. We explore the issue of conditions that may be imposed on the spacetime to yield positivity of this second term in the volume integral expression for $\mathcal{M}$.

Our construction begins with finding an appropriate generalization of the Nester 2-form [6],

$$
B^{a b}=\psi^{\dagger} \gamma^{\hat{0}} \gamma^{a b c} \nabla_{c} \psi
$$

Within the formalism, a 2-form is the correct object in the ADM case, because the boundary of a spatial hypersurface has codimension 2 with respect to the full spacetime. It is also useful to keep in mind that the ADM mass is defined in terms of the asymptotic Killing vector $\partial / \partial x^{0}$. A similar Nester 2-form was used in reference 8] to study positivity of the ADM tension of a string 7,9$]$, which is defined in terms of the asymptotic spatial Killing vector $\partial / \partial x^{1}$ tangent to the string. The Nester 2-form, in this case, differs only in the substitution of $\gamma^{\hat{1}}$ for $\gamma^{\hat{0}}$ in equation (16), corresponding to the change in asymptotic Killing vector relevant to the two different charges.

To compute the Y-ADM mass density $\mathcal{M}$ for a 1-brane, one integrates over the boundary of a co-dimension two spatial surface transverse to the brane. This boundary has co-dimension three and we therefore need to introduce a Nester 3-form. Based on the discussion above, we take this to be

$$
B^{a b c}=\psi^{\dagger} \gamma^{\hat{0} \hat{1}} \gamma^{a b c d} \nabla_{d} \psi
$$

We show below that $\mathcal{M}$ is indeed given in terms of this Nester form by

$$
\mathcal{M}=\frac{1}{8 \pi} \int_{\partial V} d s_{a} n_{b} y_{c}\left(B^{a b c}+B^{a b c *}\right),
$$

where $V$ is the co-dimension two surface with normals $n^{a}$ and $y^{a}$, as in section (IIB) above, and ${ }^{*}$ denotes complex conjugation.

We now focus on the volume integral form of $\mathcal{M}$ obtained from equation (18) using Stokes theorem 15]

$$
\mathcal{M}=\frac{1}{8 \pi} \int_{V} \sqrt{q} n_{b} y_{c} \nabla_{a}\left(B^{a b c}+B^{a b c *}\right) .
$$

It is then straightforward to show that the volume integrand above may be rewritten as the sum of two terms, as in the discsussion of the ADM mass above. The first term, which involves curvature tensors contracted with bilinears in the spinor field $\psi$, is given by

$$
\begin{aligned}
(v o l)_{1}= & R_{a b} n^{a} \xi^{b}-R_{a b} y^{a} \chi^{b}+\frac{1}{2} R \lambda \\
& -R_{a b c d} n^{a} y^{b} \xi^{c} y^{d}
\end{aligned}
$$

where $\lambda=\psi^{\dagger} \psi, \xi^{a}=-\psi^{\dagger} \gamma^{\hat{0}} \gamma^{a} \psi$ and $\chi^{a}=\frac{1}{2}\left(\psi^{\dagger} \gamma^{\hat{1}} \gamma^{a} \psi+\right.$ c.c.). The second term, which is quadratic in derivatives of the spinor field, is given by

$$
(v o l)_{2}=2 \nabla_{a} \psi^{\dagger} q^{a b} \nabla_{b} \psi-2\left(\nabla_{a} \psi^{\dagger} q_{c}^{a} \gamma^{c}\right)\left(q_{d}^{b} \gamma^{d} \nabla_{b} \psi\right)
$$

If $\psi$ is a solution to the Dirac-Witten equation $q_{b}^{a} \gamma^{b} \nabla_{a} \psi=0$, then the second term in (21) vanishes and $(v o l)_{2}$ is positive definite. The term $(v o l)_{1}$ may be simplified by choosing the spinor field $\psi$ to be an eigenvector of $\gamma^{\hat{0}}$ with eigenvalue $+i$, which is consistent with the Dirac-Witten equation. The spinor bilinears in equation (20) are then related according to $\xi^{a}=-i n^{a} \lambda$ and $\chi^{a}=y^{a} \lambda$.

Combining the two volume terms, we then have the result

$$
\begin{aligned}
\mathcal{M}= & \frac{1}{8 \pi} \int_{V}\left(\left[R_{a b} n^{a} n^{b}-R_{a b} y^{a} y^{b}+\frac{1}{2} R\right.\right. \\
& \left.\left.-R_{a b c d} n^{a} y^{b} n^{c} y^{d}\right] \psi^{\dagger} \psi+2\left(\nabla_{a} \psi^{\dagger}\right) q^{a b} \nabla_{b} \psi\right)
\end{aligned}
$$

The volume integrand can be further rewritten in a variety of ways. A compact form which is useful in subsequent calculations is

$$
(v o l)_{1}=\frac{1}{2} R_{a b c d} q^{a c} q^{b d} \psi^{\dagger} \psi
$$

This version highlights the similarities with the codimension one case, see Section VI.

A second form that will also be useful is

$$
\begin{aligned}
\mathcal{M}=\frac{1}{8 \pi} \int_{V}[ & \left(G_{a b} n^{a} n^{b}-R_{a b c d} y^{a} y^{c} q^{b d}\right) \psi^{\dagger} \psi \\
& \left.+2\left(\nabla_{a} \psi^{\dagger}\right) q^{a b} \nabla_{b} \psi\right]
\end{aligned}
$$

Here, after making use of Einstein's equation, the first term is simply equal to the energy density $T_{a b} n^{a} n^{b}$, which supports the idea that the Y-ADM charge $\mathcal{M}$ is a kind of mass per unit length. It is less clear how one should interpret the Riemann tensor term. Since it is not directly related to the stress energy, one cannot as in the case of the ADM mass and tension, ensure positivity of $\mathcal{M}$ by imposing an energy condition. However, the fact that the spinor contribution, as in the ADM case, is positive definite suggests that one look for additional conditions on brane spacetimes such that the Riemann term is also positive. We turn to this issue in Section $\nabla$ below. First, we return to the issue of the spinor boundary integral in equation (18). 


\section{A. Further Study of the Spinor Boundary Term}

In this section, we demonstrate that the spinor boundary integral in equation (18) does indeed reproduce the definition of the Y-ADM mass density given in section III We also include a further analysis of the Y-ADM boundary integral, which shows that the Y-ADM mass density $\mathcal{M}$ depends only on the metric $q_{a b}$ on the surface transverse to the brane. This result provides further motivation for regarding the mass density $\mathcal{M}$ as an intrinsic (rather than extensive) property of the brane.

We consider transverse asymptotically flat spacetimes. The metric therefore approaches the Minkowski metric $\eta_{a b}$ at spatial infinity, and we can assume that the spinor field $\psi$ approaches one of the constant spinors $\psi^{(0)}$ of flat spacetime - i.e. satisfying $\bar{\nabla}_{a} \psi^{(0)}=0$. The Nester 3 -form can then be schematically rewritten as $B[\nabla]=B[\nabla-$ $\bar{\nabla}]+B[\bar{\nabla}]$. One can check that $B[\bar{\nabla}]$ vanishes sufficiently fast as $\psi$ approaches $\psi^{(0)}$ that the boundary term just depends only on the difference of the derivative operators. One can then show that $\left(\nabla_{a}-\bar{\nabla}_{a}\right) \psi=-\frac{1}{4} \nabla_{[m} h_{n] a} \gamma^{m n} \psi$, with the result that the boundary integrand becomes

$$
\begin{aligned}
n_{a} x_{b}\left(B^{a b c}\right. & + \text { c.c. })= \\
& \frac{1}{4} \nabla_{[m} h_{n] d} q_{a}^{c} q_{b}^{d} \psi^{\dagger}\left(\gamma^{a b} \gamma^{m n}+\gamma^{m n \dagger} \gamma^{a b}\right) \psi .
\end{aligned}
$$

Here we have replaced $\gamma^{a b \dagger}$ by $\gamma^{a b}$ since it is projected by the spatial metric $q_{a b}$. Except for $h_{a b}$, all quantities in equation (25) are background quantities. Depending on whether the coordinates $x^{m}, x^{n}$ are timelike or spacelike, either the commutator or the anticommutator of the gamma matrices in (25) contributes. The asymptotically flat boundary conditions (4) imply that terms involving $h_{x i}$ or $h_{t i}$, where the indices $i, j$ are projected by $q_{i}{ }^{j}$, are higher order. It is then straightforward to check that the only terms which contribute to the sum in equation (25) are those with $m, n=i, j$. We then have

$$
\mathcal{M}=\frac{1}{8 \pi} \int_{\partial V} d s_{a} q^{a d} q^{c b} \nabla_{[b} h_{d] c}
$$

which agrees with the expression in section (III).

We have seen that the spinor formalism of this section gives an alternate construction of Y-ADM charges. The original formulation [1] based on the AD construction 3] has the merit of establishing a clear relationship between the Y-ADM charges and the Killing-Yano tensors of the background flat spacetime. However, the volume integral form of the charge, in this construction, involves only the linearized curvature tensors around the flat background. The strength of the spinor construction is that it yields a volume integral expression that depends on the exact curvature tensor of the spacetime together with a positive definite spinor term, and is much more useful in trying to assess positivity properties.

We finish this section by rewriting the Y-ADM mass density $\mathcal{M}$ in terms of the transverse metric perturbation $\delta q_{a b}$ defined near spatial infinity via the relation $q_{a b}=$ $\delta_{a b}+\delta q_{a b}$. Again making use of the assumption that $h_{t i}$ and $h_{x i}$ are of higher order at infinity, we find that in the boundary integral (26) we may write $q_{a}^{b} q^{c d} q_{n}^{m} \nabla_{b} h_{m c}=$ $D_{a} \delta q_{n}^{d}$, where $D_{a}$ is the flat covariant derivative operator on the slice $V$. The formula for $\mathcal{M}$ then becomes

$$
\mathcal{M}=\frac{1}{8 \pi} \int_{\partial V} d s_{a}\left(D_{b}\left(\delta q_{c d} q^{a c} q^{b d}\right)-D^{a} \delta q_{b c} q^{b c}\right) .
$$

This expression highlights the fact that $\mathcal{M}$ is an intrinsic quantity on the codimension-two volume $V$, since it only depends on $q_{a b}$. Also note that if instead $V$ was a codimension-one volume, and $q_{a b}$ the metric on $V$, then equation (27) gives the usual expression for the ADM mass.

\section{POSITIVITY}

We have seen above that the volume integral expression for the Y-ADM mass density $\mathcal{M}$, coming from a rank two Killing-Yano tensor, differs from the ADM mass, which comes from a rank one Killing-Yano tensor, in that the Riemann tensor contributes to the volume integrand, as well as Ricci and scalar curvature terms which occur in both cases. These latter terms can be related to the stress energy tensor via the Einstein equation, so that positivity, in the ADM mass case, can be phrased in terms of energy conditions. The prospects for positivity of the Y-ADM mass density $\mathcal{M}$ therefore look less favorable. However, the fact that the spinor field makes a positive definite contribution to $\mathcal{M}$, suggests that we look for conditions under which the Reimann tensor term is positive as well. In this section we derive positivity results for the Y-ADM mass density $\mathcal{M}$ under two kinds of conditions; first, by requiring conditions on the Weyl tensor of the spacetime, and second, in the case of exact translational symmetry in the $x^{1}$-direction.

\section{A. Conditions on the Weyl Tensor}

The idea here is to decompose the Reimann tensor into trace pieces, the Ricci and scalar contributions, and the rest, the Weyl tensor. When the Weyl term vanishes, the remaining terms in the volume integrand can be related to the stress-energy, which leads to an energy condition for positivity of $\mathcal{M}$. In $D$ dimensions, the decomposition of the Riemann tensor is given by

$$
\begin{aligned}
R_{a b c d}= & C_{a b c d}+\frac{2}{(D-2)}\left(g_{a[c} R_{d] b}-g_{b[c} R_{d] a}\right) \\
& +\frac{2}{(D-1)(D-2)} R g_{a[c} g_{d] b}
\end{aligned}
$$

Substitution into equation (20) then yields

$$
\begin{aligned}
(v o l)_{1}= & {\left[\frac{D(D-3)}{2(D-1)(D-2)} R+\frac{D-3}{D-2} R_{a b}\left(n^{a} n^{b}-y^{a} y^{b}\right)\right.} \\
& \left.-C_{a b c d} n^{a} y^{b} n^{c} y^{d}\right] \psi^{\dagger} \psi
\end{aligned}
$$


One simple condition to consider is the vanishing of the Weyl tensor, $C_{a b c d}=0$. It is then clear that an appropriate energy condition would assure positivity of $(\mathrm{vol})_{1}$.

Another condition under which the Weyl tensor term in equation (29) vanishes is when the metric is algebraically special with all four principal null directions coinciding, and the null vector lies in the plane tangent to the brane. Explicitly, this means assuming that $p^{a} C_{a b c d}=0$ for a null vector $p^{a}=n^{a} \pm y^{a}$. To see this, define the vectors $k^{a}=n^{a}+y^{a}$ and $q^{a}=n^{a}-y^{a}$, which allows us to write

$$
C_{a b c d} n^{a} y^{b} n^{c} y^{d}=\frac{1}{4} C_{a b c d} k^{a} q^{b} k^{c} q^{d} .
$$

If either $k^{a}$ or $q^{a}$ is the principal null vector $p^{a}$, then this term vanishes. Note that while either this conditions or comformal flatness is sufficient to make the Weyl tensor term in equation (29) vanish, but is more than is needed. It would be interesting to find a geometrical condition which was minimal in the sense of being both necessary and sufficient.

If the Weyl tensor term vanishes, then $(\mathrm{vol})_{1}$ can be rewritten in terms of the stress energy tensor using Einstein's equation. If we make the definitions $\rho=T_{a b} n^{a} n^{b}$, $p_{\hat{y}}=T_{a b} y^{a} y^{b}$ and similar definitions for the pressures $p_{\hat{i}}$ in directions transverse to the string, then the volume integral expression for $\mathcal{M}$ becomes

$$
\begin{aligned}
\mathcal{M}= & \frac{(D-3)}{(D-1)} \int_{V}\left[\left(\rho-p_{\hat{y}}+\frac{1}{D-2} \sum_{i} p_{\hat{i}}\right) \psi^{\dagger} \psi\right. \\
& \left.+2\left(\nabla_{a} \psi^{\dagger}\right) q^{a b} \nabla_{b} \psi\right]
\end{aligned}
$$

where the index $i$ runs over the transverse coordinates. If we therefore have any set of condition, such as those suggested above, which lead to the vanishing of the Weyl tensor term in (29), then we have shown that the Y$\mathrm{ADM}$ mass density $\mathcal{M}$ is positive if the additional energy condition

$$
\left(\rho-p_{\hat{y}}+\frac{1}{D-2} \sum_{i} p_{\hat{i}}\right) \geq 0
$$

is satisfied [16].

The energy condition (32) has a straightforward physical interpretation. The quantity $-p_{\hat{y}}$ is simply the tension along the string, and equation (31) implies that this tension contributes positively to $\mathcal{M}$. Note that for a standard cosmic string we have $p_{\hat{y}}=-\rho$. The pressures $p_{\hat{i}}$ in the directions transverse to the string contribute as they do for a particle-type configuration - positive pressures make a positive contribution to the mass. Note also that in the non-relativistic limit, where the pressures are negligible compared to the energy density, equation (31) implies that $\mathcal{M}$ is the energy density integrated over a transverse slice, plus a positive definite contribution from the gravitational field.

\section{B. Translational Symmetry and Kaluza-Klein Reduction}

Suppose that translation along the string is a actually a symmetry of the $D$-dimensional spacetime. One would then expect that there is a $(D-1)$-dimensional point of view, in which the Y-ADM mass density $\mathcal{M}$ is related to the $(D-1)$-dimensional ADM mass.

We will need the following Gauss-Codazzi relation for a codimension one submanifold with unit normal vector $w^{a}$. If we write the metric as

$$
g_{a b}=s_{a b}+(w \cdot w) w_{a} w_{b},
$$

where $w \cdot w= \pm 1$ depending on whether the normal $w^{a}$ spacelike or timelike, then the Riemann tensors of $g_{a b}$ and $s_{a b}$ are related according to

$$
\begin{aligned}
s_{a}^{m} s_{b}^{n} s_{c}^{r} s_{d}^{p} R_{m n r p}[g]= & R_{a b c d}[s] \\
& +(-w \cdot w)\left(J_{a c} J_{b d}-J_{a d} J_{b c}\right)
\end{aligned}
$$

where $J_{a b}=s_{a}^{c} \nabla_{c} w_{b}$ is the extrinsic curvature of the submanifold.

Take the normal vector to be $y^{a}$, the direction tangent to the string. The metric $s_{a b}$ is then Lorentzian. The volume integrand for $\mathcal{M}$ can be expressed in terms of the Einstein tensor for a $(D-1)$-dimensional metric $G_{a b}^{(D-1)}[s]$ as follows. Making use of equation (34) one finds that

$$
\begin{aligned}
(v o l)_{1} & =\frac{1}{2} q^{a c} q^{b d} R_{a b c d}[g] \\
& =\frac{1}{2} q^{a c} q^{b d}\left(R_{a b c d}[s]+J_{a c} J_{b d}-J_{a d} J_{b c}\right) \\
& =G_{a b}^{(D-1)}[s] n^{a} n^{b}+\frac{1}{2}\left(\beta_{a b} \beta^{a b}-\beta^{2}\right)
\end{aligned}
$$

where $\beta_{a b}=q_{a}{ }^{m} q_{b}{ }^{n} \nabla_{m} y_{n}$ is the projection of the extrinsic curvature $J_{a b}$ onto the directions transverse to the string.

We now assume that the spacetime has a spatial translation Killing field $V^{a}$ that is parallel to the brane. Near infinity, the Killing vector $V^{a}$ approaches $\partial / \partial x^{1}$, which also coincides with the unit normal vector $y^{a}$ in this limit. Further assume that we extend $y^{a}$ into the interior of the spacetime, such that it is parallel to the Killing field $V^{a}$, i.e. let $V^{a}=F y^{a}$ where $V_{a} V^{a}=F^{2}$. We then have $J_{a b}=0$, since $J_{a b}=(1 / 2) £_{V} s_{a b}$. From the last line of equation (35), we then have the result

$$
\mathcal{M}=\frac{1}{8 \pi} \int_{V}\left(n^{a} n^{b} G_{a b}^{(D-1)}[s] \psi^{\dagger} \psi+2\left(\nabla_{a} \psi^{\dagger}\right) q^{a b} \nabla_{b} \psi\right)
$$

At this point one needs to know something about the dimensionally reduced theory [17], in order to draw a conclusion about the Y-ADM mass density $\mathcal{M}$. If the dimensionally reduced Einstein tensor is equal to a stress energy which satisfies the dominant energy condition, then $\mathcal{M}$ is positive. Indeed, the form of the boundary term 
given in equation (27) is the standard ADM mass for the metric $s_{a b}$. Therefore, the argument leading to (36) gives a consistency check on the meaning of the mass per unit length: when dimensional reduction is possible, the mass per unit length coincides with the ADM mass of the lower dimensional theory.

\section{A GENERALIZED HAMILTONIAN FOR TRANSVERSE ASYMPTOTICALLY FLAT SPACETIMES?}

In this final section we point out that the form of the volume integral expression for $\mathcal{M}$ derived in section IV suggests that the term $(\mathrm{vol})_{1}$ might serve as a generalized Hamiltonian for the evolution for transverse asymptotically flat brane spacetimes. By generalized Hamiltonian evolution we mean specifying data on a codimension 2 slice transverse to the brane and evolving the data via a system of first order PDE's in the directions tangent to the brane. In this view, we think of ordinary Hamiltonian evolution as specifying data on a codimension 1 slice transverse to the worldline of particle-like sources and then evolving in the single direction tangent to the particle worldline.

First recall that in the codimension 1 case, if we write $g_{a b}=-n_{a} n_{b}+l_{a b}$, then the Einstein tensor term in the volume integrand is simply given by

$$
\begin{aligned}
(v o l)_{1} & =\left(G_{a b} n^{a} n^{b}\right) \psi^{\dagger} \psi \\
& =\frac{1}{2}\left(R_{a b c d} l^{a c} l^{b d}\right) \psi^{\dagger} \psi
\end{aligned}
$$

If we then change to Hamiltonian variables we have

$$
(v o l)_{1}=\frac{1}{2}\left(R[l]-k_{a b} k^{a b}+k^{2}\right) \psi^{\dagger} \psi
$$

where $R[l]$ is the scalar curvature of the $D-1$ dimensional metric $l_{a b}$ and $k_{a b}=l_{a}^{c} l_{b}{ }^{d} \nabla_{c} n_{d}$ is the extrinsic curvature of the slice with normal $n_{a}$. The factor multiplying $\psi^{\dagger} \psi$ is, of course, simply proportional to the gravitational Hamiltonian. An additional momentum term would also arise if $\psi$ were not taken to be an eigenvector of $\gamma^{\hat{0}}$.

In the codimension 2 case addressed in this paper, it is interesting that the volume term $(\mathrm{vol})_{1}$ has a very similar form to equation (38) and may be similarly rewritten in
Hamiltonian form,

$$
\begin{aligned}
(v o l)_{1} & =\frac{1}{2}\left(R_{a b c d} q^{a c} q^{b d}\right) \psi^{\dagger} \psi \\
& =\frac{1}{2}\left(R[q]-K_{a}^{a e} K_{a e}^{a}+K_{a b}^{e} K_{e}^{a b}\right) \psi^{\dagger} \psi
\end{aligned}
$$

It seems natural to speculate that this quantity, along with the analogous momentum terms that would appear if we did not take $\psi$ to be an eigenvector of $\gamma^{\hat{0}}$, play a role in a generalized Hamiltonian evolution of brane spacetimes of the sort described above. We note, as well, that extrinsic curvature can be further decomposed as $K_{a b}^{c}=\alpha_{a b} n^{c}+\beta_{a b} y^{c}$, where $\alpha_{a b}=q_{a}^{c} q_{b}^{d} \nabla_{c} n_{d}$ and $\beta_{a b}=$ $-q_{a}^{c} q_{b}^{d} \nabla_{c} y_{d}$. We can then write

$$
\begin{aligned}
\frac{1}{2} R_{a b c d} q^{a c} q^{b d}= & \frac{1}{2}\left(R[q]-\alpha_{a b} \alpha^{a b}+\alpha^{2}\right. \\
& \left.+\beta_{a b} \beta^{a b}-\beta^{2}\right),
\end{aligned}
$$

where all contractions are done with the positive definite metric $q_{a b}$. We can thus one can read off whether the extrinsic curvature terms are positive or negative.

It is also interesting to compare equation (40) to the Hamiltonian in the double null formalism developed by Hayward 12. In certain gauge conditions, equation (40) is identical with the Hamiltonian in double null formalism, at least in four dimensional spacetimes (see reference [13]). The extension to higher dimensional spacetimes is straightforward. Since we focus on the codimension 2 integral manifolds, the twist term also vanishes. Therefore, we can realise that equation (40) is indeed the Hamiltonian. This is a natural consequence because the double null formalism provides us with a codimension 2 space normal to two null directions.

Acknowledgements: The work of DK and JT was supported in part by National Science Foundation grant NSF PHY0244801. The work of TS was supported by Grantin-Aid for Scientific Research from Ministry of Education, Science, Sports and Culture of Japan(No.13135208, No.14740155 and No.14102004). The work of ST was supported by the 21st Century COE Program at TokyoTech "Nanometer-Scale Quantum Physics" supported by the Ministry of Education, Culture, Sports, Science and Technology.
[1] D. Kastor and J. Traschen, "Conserved gravitational charges from Yano tensors," JHEP 0408, 045 (2004) arXiv:hep-th/0406052.

[2] K. Yano, "Some Remarks on Tensor Fields and Curvature," Ann. Math. 55, 328 (1952).

[3] L. F. Abbott and S. Deser, "Stability Of Gravity With A Cosmological Constant," Nucl. Phys. B 195, 76 (1982).

[4] R. Schoen and S. T. Yau, "On The Proof Of The Positive
Mass Conjecture In General Relativity," Commun. Math. Phys. 65, 45 (1979).

[5] E. Witten, "A Simple Proof Of the Positive Energy Theorem," Commun. Math. Phys. 80, 381 (1981).

[6] J. Nester, Phys. Lett. 83A, 241(1981).

[7] P. K. Townsend and M. Zamaklar, "The first law of black brane mechanics," Class. Quant. Grav. 18, 5269 (2001) arXiv:hep-th/0107228. 
[8] J. H. Traschen, "A positivity theorem for gravitational tension in brane spacetimes," Class. Quant. Grav. 21, 1343 (2004) arXiv:hep-th/0308173.

[9] J. H. Traschen and D. Fox, "Tension perturbations of black brane spacetimes," Class. Quant. Grav. 21, 289 (2004) arXiv:gr-qc/0103106.

[10] S. W. Hawking and G. T. Horowitz, "The Gravitational Hamiltonian, action, entropy and surface terms," Class. Quant. Grav. 13, 1487 (1996) arXiv:gr-qc/9501014.

[11] G. W. Gibbons, S. W. Hawking, G. T. Horowitz and M. J. Perry, "Positive Mass Theorems For Black Holes," Commun. Math. Phys. 88, 295 (1983).

[12] S. A. Hayward, Class. Quantum Grav. 10, 779(1993).

[13] S. A. Hayward, "Quasilocal gravitational energy," Phys. Rev. D 49, 831 (1994) arXiv:gr-qc/9303030.

[14] In this paper we take the $p$ spatial directions tangent to the brane will be taken to be either infinite in extent or periodically identified on a torus. It might also be interesting to consider wrapping the tangent directions on nontrivial cycles of more general reduced holonomy spaces.

[15] We assume that there are no horizons in the spacetime so that no inner boundary terms arise in applying Stokes theorem. An extension of these results to black brane spacetimes with horizons would presumably require additional boundary conditions on the spinor field $\psi$ to eliminate inner boundary terms, as in reference [1].

[16] This energy conditions differs from standard energy conditions, such as the dominant energy condtion, in that it refers to the normal vectors in a given slicing of the spacetime, rather than a general class of vectors.

[17] Note that the metric $s_{a b}$ is not rescaled by a dilaton factor, as it would be in the Kaluza-Klein ansatz, and that no Kaluza-Klein gauge fields appear because we have chose the normal direction to be parallel to the Killing vector $v^{a}$. Therefore, in these coordinates, the shift terms $g_{y t}$ and $g_{y I}$ vanish. 